American Journal of Pharmaceutical Education 2019; 83 (10) Article 7099.

\title{
COMMENTARY
}

\section{Enhancing Educational and Leadership Opportunities for Second-Year Pharmacy Residents}

\author{
Lauren Fruhling, PharmD, Michelle LaFever, PharmD, Brian Erstad, PharmD \\ University of Arizona College of Pharmacy, Tucson, Arizona
}

Submitted March 29, 2018; accepted August 27, 2019; published December 2019.

\begin{abstract}
Leadership is a critical aspect of residency training; however, little guidance is provided in the literature regarding practical implementation of leadership training within residency programming. Programming was developed within a second-year (PGY-2) critical care residency program to meet the educational and leadership needs of the residents. The format of the meetings has evolved over time from a journal club-style format to an interdisciplinary format discussing far more than literature. With the residents taking the lead in the biweekly educational sessions, there is great opportunity for leadership development. Similar programming has the potential to strengthen residency experiences for residents in other programs.
\end{abstract}

Keywords: residency, education, leadership, pharmacy

\section{INTRODUCTION}

Pharmacy residency training is an evolving field with various organizations committed to development of appropriate residency training standards. Topics such as clinical education and leadership are important areas of development for residents during their training. The American Society of Health-System Pharmacists (ASHP), which accredits pharmacy residencies, recently published a report entitled "Leadership as a Professional Obligation," in which clinical and leadership skills are encouraged to be taught through formal education. ${ }^{1}$ Furthermore, ASHP accreditation standards (competency area R3: Leadership and Management for PGY-2 Critical Care, and R1: Demonstrate leadership and practice management skills for PGY-2 Emergency Medicine) also comment on the importance of residency programs having a leadership component in addition to clinical training. ${ }^{2,3}$ One study observed that $76.2 \%$ of surveyed pharmacy residents from 2011 to 2014 spent less than $20 \%$ of their residency training focused on leadership. ${ }^{4}$ Despite the importance of leadership within the pharmacy profession, little guidance is provided for practical implementation of residency programming to develop pharmacy leaders. Additionally, with healthcare adopting a layered learning practice model, opportunities for pharmacy residents to adopt a leadership role may not be as clear. ${ }^{5}$ Years after developing and initiating a critical care pharmacy service as well as the PGY-2 Critical Care

Corresponding Author: Lauren Fruhling, 1501 N Campbell Ave., \#5505, Tucson, AZ 85724. Tel: 520-444-1164. Email: lfruhling@renown.org. residency program, the clinical faculty member who was residency program director at the time sought to improve PGY-2 training and provide an opportunity for PGY-2 leadership within the program. We describe a best practice solution, entitled "Erstad Hour," that was developed in part to foster the leadership component of the critical care pharmacy resident experience.

The inception of Erstad Hour occurred near the start of 2000 when first-year (PGY-1) and PGY-2 pharmacy residents on the medical and surgical ICU services began to have scheduled meetings with the critical care residency program director to discuss important articles pertaining to critical care practice with an emphasis on critical care pharmacotherapy. This programming was intended to meet an educational void within the critical care rotations, as the service was often too hectic for regular journal club discussions. To start, at the beginning of PGY-2 year or the PGY-1 critical care rotation each resident was provided with a list of key readings frequently encountered in general medical/surgical ICU settings. During Erstad Hour sessions, this list of readings was expanded to include reviews of key articles and guidelines assigned to each resident as part of the twice weekly meetings. Residents were expected to perform indepth reviews of their assigned article(s) and be ready to present the findings in approximately five minutes, as the intent was primarily to familiarize all participants with the paper so an active discussion could take place. The resident presenting the article was expected to serve as a content expert and answer questions during the discussion with the help of the residency program director and other 


\section{American Journal of Pharmaceutical Education 2019; 83 (10) Article 7099.}

preceptors in attendance. The overall purpose of Erstad Hour, as stated in the residency manual, was to augment and compliment learning associated with direct patient care activities in critical care clinical settings. At the time of its origin, Erstad Hour was more of a traditional journal club, although even then there was a deliberate attempt to expose the PGY-2 critical care resident to all key articles, including clinical practice guidelines, during the residency year. Summaries of the compiled key articles were subsequently published by the residency program director, with a PGY-1 and a PGY-2 pharmacy resident serving as coauthors. ${ }^{6}$ The original article compilation reflected the perspective of the program's director, but subsequent revisions of the paper were authored by the residency program director with other clinical pharmacists from a diverse range of specialty and sub-specialty critical care practice settings. ${ }^{7,8}$

\section{Evolution of the Erstad Hour}

Erstad Hour evolved organically throughout the years to meet multiple needs of the residency program. During the formative years of Erstad Hour, the residency program director allowed incoming PGY-2 critical care residents to experiment with the article assignments. For example, during a couple of residency years there was a deliberate decision by the critical care residents to discuss all papers published in the most current versions of the key papers and guidelines reviews. This meant that many of the PGY-1 residents had minimal say in their assigned readings, which in some cases was met with some resistance. Over time the critical care residents continued to try to cover the key papers during the year, but allowed for input by PGY-1 residents on critical care rotations. At times, this meant discussions of the same paper more than once a year. By 2005, the residency program director had developed expected outcomes, program-specific goals and objectives for the year-long Erstad Hour program, which were compiled by way of Bloom's taxonomy. ${ }^{9}$ Feedback of resident performance in Erstad Hour sessions is provided by the facilitating preceptor to the respective program directors and preceptors. This feedback is then provided to residents during final feedback discussions with the preceptor as well as documented within the program's electronic evaluation program as formal feedback. For the residents that organize the Erstad Hour sessions, feedback is provided during quarterly meetings with the respective program director as well as documented on the program's electronic evaluation program as formal feedback for a longitudinal activity.

Another evolution within Erstad Hour occurred after the residency program director participated in the development of a new accreditation guideline by ASHP for
PGY-2 critical care residencies that were endorsed by the Society of Critical Care Medicine. The original set of educational goals and objectives had not been revised since 1997. The new accreditation guideline that was approved by the ASHP Commission on Credentialing and the ASHP Board of Directors in 2007 had an appendix intended to enhance residents' understanding and appreciation of the implications of medication therapy. The guideline was divided into three sections: Organ-System Related, Specific Considerations, and Supportive Care. ${ }^{10}$ For a number of years following its publication, this appendix was used by the residency program director and PGY-2 critical care residents to help inform the choice of articles for discussion in Erstad Hour. The accreditation guideline was further updated in 2017.

\section{Current Practice}

Erstad Hour has been shaped based on past experiences of previous residents. Currently, the PGY-2 critical care and PGY-2 emergency medicine residents meet twice weekly for approximately a one-hour session to present and discuss current literature as was done in past years prior to the development of our emergency medicine residency. In addition to critical care PGY-2 residents, PGY-2 residents from other specialties, PGY-1 residents, and pharmacy students also attend when they are on critical care rotations. Erstad Hour meetings are considered protected academic time to ensure attendance and appropriate discussion. To encourage active participation by all residents, prior to the start of the rotation block, an email is sent out to all participants to identify if there are any topics the incoming residents would like to discuss. Besides suggestions from residents, topics can be chosen based off landmark critical care trials or suggestions from preceptors. Another factor that affects article choice is a list of topics put together by the residency program director that should be discussed throughout the residency year. Erstad Hour allows the opportunity to fit in topics that might have not been discussed at length on rotations. Prior to the Erstad Hour session, the designated PGY-2 resident leader for the session puts together a list of four or five articles that will be discussed and assigns the articles to residents. These articles are placed in an online file storage system so all attending residents have long-term access to all articles discussed in that session. Besides residents, all critical care and emergency medicine preceptors are invited to attend to encourage preceptor participation and as an opportunity for residents to learn from diverse preceptor experiences. Currently, each session is limited to four to five pertinent articles to ensure meaningful article selection and ample discussion time regarding each article and for group discussion after 


\section{American Journal of Pharmaceutical Education 2019; 83 (10) Article 7099.}

all the articles are presented. This also allows for the feasibility of each resident to read each included article to maximize discussion and overall learning for each session. Beyond limiting the number of articles, the lead residents also encourage article presentations to be approximately five to seven minutes in length to encourage a brief yet thorough presentation of the article. During or at the end of Erstad Hour, it is not uncommon for the faculty leader, residency program directors, or preceptors to comment on the choice of articles selected for discussion by the resident. More important, or other important papers, may be mentioned for reference or future discussions. Although article topic discussions are the mainstay of Erstad Hour, there are other teaching opportunities that have been employed in recent years to provide other education opportunities.

A special event we have been able to facilitate is the incorporation of interdisciplinary teaching by other medical professionals, as well as a chance for other pharmacy preceptors to teach about their specialty or subspecialty practice. For example, for one of our sessions a bedside discussion of ventilator management was given by a pulmonary/critical care medical fellow. This fellow took the hour to explain the basics of the ventilator and important points to look for when assessing patient tolerance of the ventilator. Following the bedside discussion, the fellow then did a whiteboard teaching session on ventilator modalities and the appropriate uses for each one. Additional teachings that have been done by other members of the healthcare team include advanced cardiac life support (ACLS) management beyond the algorithm, management of anticoagulation by rotational thromboelastography (ROTEM), and emergent anticoagulation reversal. Each of these unique educational sessions has been conducted by either medical or pharmacy specialists in their respective fields to provide both interdisciplinary education and a chance for preceptors to provide education regarding special topics. To provide additional leadership opportunities, these lectures are also often delivered by the PGY-2 residents organizing the sessions. Based on feedback provided to the organizing resident following sessions, these specialty sessions have been extremely well received within the critical care department and attended by both residents and pharmacists who have an interest in these specialized topics. The goal is to hold at least one of these interdisciplinary sessions for each rotating resident group, occurring every four to five weeks, although this is subject to schedules and availability.

An additional special event that we are able to facilitate is the review of grant proposals and articles written for submission to journals. As the faculty leader is approached to review articles and grants, he asks permission to include the Erstad Hour group in this process. Upon approval, the items are de-identified, and all journal, financial, and/or author information is removed to maintain strict confidentiality. Articles are provided to the group on how to perform quality reviews. The group reviews the information and prepares a summary of their thoughts regarding the quality of article for publication or the merits of grant proposals. At times with the permission of the editor, PGY-2 residents are offered the opportunity to participate as a co-reviewer with the faculty leader to gain a deeper understanding of the peer-review process. This is not only a unique learning opportunity, but it also provides the residents with the opportunity to get connected with various journals as an article reviewer going forward in the resident's career.

\section{Implementation in Other Programs}

The development of similar programming has the potential to strengthen learning experiences for various learning environments. While Erstad Hour was initially developed specifically for critical care pharmacy residents at our institution, it could easily be adapted for a multitude of specialty practices, topic areas, and practice settings, just as it has been expanded at our institution to include emergency medicine residents. First steps towards implementation of similar programming would be to identify a residency program director or preceptor leader and determine which students or residents should organize the sessions. The academic and professional goals of the group should be discussed and agreed upon. Independence of the learners to develop topics may vary based upon ability and institution preference; however, increased learner independence adds to the leadership potential. Depending on the involvement of the residency program director or preceptor leader in the pharmacy community, alternative programming such as article reviews may be added. The educational opportunities possible with an Erstad Hour-style programming can be tailored based upon personnel, practice setting, and group goals.

\section{CONCLUSION}

Leadership opportunities may be a challenging aspect of ASHP accreditation standards in which to demonstrate full compliance. Implementation of programming similar to Erstad Hour has the potential to improve the residency training experience, particularly in the area of leadership.

\section{REFERENCES}

1. American Society of Health-System Pharmacists statement on leadership as a professional obligation. Am J Health Syst Pharm. 2011.1;68(23):2293-2295.

2. American Society of Health-System Pharmacists. Required competency areas, goals, and objectives for postgraduate year two (PGY2) critical care pharmacy residencies. 2016. 


\section{American Journal of Pharmaceutical Education 2019; 83 (10) Article 7099.}

3. American Society of Health-System Pharmacists. Educational outcomes, goals, and objectives for postgraduate year two (PGY2) pharmacy residencies in emergency medicine. 2012.

4. Cho JC, Girnys JP. National survey regarding the importance of leadership in PGY1 pharmacy practice residency training. Hosp Pharm. 2015;50(11):978-984.

5. Loy BM, Yang S, Moss JM, Kemp DW, Brown JN. Application of the layered learning practice model in an academic medical center. Hosp Pharm. 2017;52(4):266-272.

6. Erstad BL, Jordan CJ, Thomas MC. Key articles and guidelines relative to intensive care unit pharmacology. Pharmacotherapy. 2002;22:1594-1610.
7. Erstad BL, Martin SJ, Brophy GM, et al. Key articles and guidelines relative to intensive care unit pharmacology - 2004 . Pharmacotherapy. 2005;25:585-610.

8. Erstad BL, Brophy GM, Martin SJ, et al. Key articles and guidelines relative to intensive care unit pharmacotherapy: 2009 Update. Pharmacotherapy. 2005;25:585-610.

9. Bloom BS. Taxonomy of Educational Objectives, Handbook I: The Cognitive Domain. New York: David McKay Co Inc; 1956. 10. American Society of Health-System Pharmacists. Educational Outcomes, Goals, and Objectives for Postgraduate Year Two (PGY2) Pharmacy Residencies in Critical Care. American Society of HealthSystem Pharmacists. 2007. 